\title{
Study on the Relationship Between Energy Storage Efficiency and Charg- ing Mode of Super Capacitor
}

\author{
Zhang Dedi ${ }^{*}$
}

College of Electric and Electronic Engineering, Zibo Vocational Institute, Zibo, 255314, China

\begin{abstract}
Super capacitor is now widely used in the field of design and daily life. Super capacitor is different from the normal battery, it occupyies the seat as an important role in creasing in energy storage area due to its own advantages. The principle and applications of the super capacitor were described in this article, and the equivalent circuit model of the super capacitor was modified and given. The definition of charging efficiency, discharging efficiency, storage efficiency and energy conversion efficiency were given out clearly. We can concluded that the charging efficiency of constant voltage is low and not suitable for charging through analyses of the three charging ways of constant-current, constant-voltage and constant-power. The constant current charging is fast and efficient, the constant power charging can only suit for special field as photovoltaic power generation.
\end{abstract}

Keywords: Super capacitor, energy storage, charging efficiency, charging mode.

\section{INTRODUCTION}

Super Capacitor is a kind of new type energy storage device between traditional capacitor and battery which also named Electric Double Layer Capacitor (EDLC) or Power Capacitor. It has both characteristics of rapid charge and discharge of capacitor, and also have battery energy storage features. Super capacitor is via an external electric field polarization electrolyte, and produce positive and negative ions in the electrolyte, and adsorb on the surface plate, then realize the energy storage. In recent years, the super capacitor research in the world gradually increased, and achieved certain results. There are a lot of super capacitor of different company to enter the market [1-3].

Super capacitors have many advantages compared with other batteries. Such as it have high energy density, charging and discharging quickly, long cycle life, high efficiency of storage, pollution-free, it also has wide working temperature, high reliability, and can charge and discharge with rapid circulation and discharge characteristics for long time. Super capacitor is mainly used in UPS, cars, ignition, flash and other fields, especially in the development of application in the field of electric vehicles has aroused extensive attention of the world. Super capacitor energy storage has its own characteristics such as charging efficiency and charging ways has a lot to do, influenced by temperature, cycle times. In some larger load of transformer substation with the method of super capacitor and battery hybrid energy storage, play their respective advantages, improve efficiency and cost savings. This paper analyzes the super capacitor charging efficiency, discharge efficiency and energy storage efficiency, and research the affect on the charging efficiency and charging way $[4,5]$.

Principle of energy storage of super capacitor is different from the battery, its capacity of the process of charge and discharge states has its own characteristics. Super capacitor charging and discharging efficiency is mainly influenced by charge and discharge current, temperature, charge and discharge cycles and other factors such as including charge and discharge flow. The super capacitor generally adopt the method of constant current charging and voltage limiting, in addition, also has constant rate of word charging. Constant current charging with voltage limiting method for control of the highest voltage of $U_{\max }$, constant current charging into constant pressure after floating, until the super capacitor is fully charged [6-8]. The advantage of using this charging method: charging with the larger current to save the charging time at the beginning, later the constant-voltage charging can reach the end of the charging by small current charging, not only ensure full, also can avoid the super capacitor internal high temperature and the effect on the characteristic of super capacitor capacity.

\section{THE ANALYSIS OF ENERGY STORAGE AND EF- FICIENCY OF SUPER CAPACITOR}

\subsection{The Energy Storage Principle and Equivalent Circuit of Super Capacitor}

Super capacitor generally divided into electric double layer capacitor and faraday capacitor, in this article we will discuss electric double layer capacitor (EDLC). The electrode mainly made with carbon material including the electrolyte, the electrodes and the separator. The structure is shown in Fig. (1) $[9,10]$. 


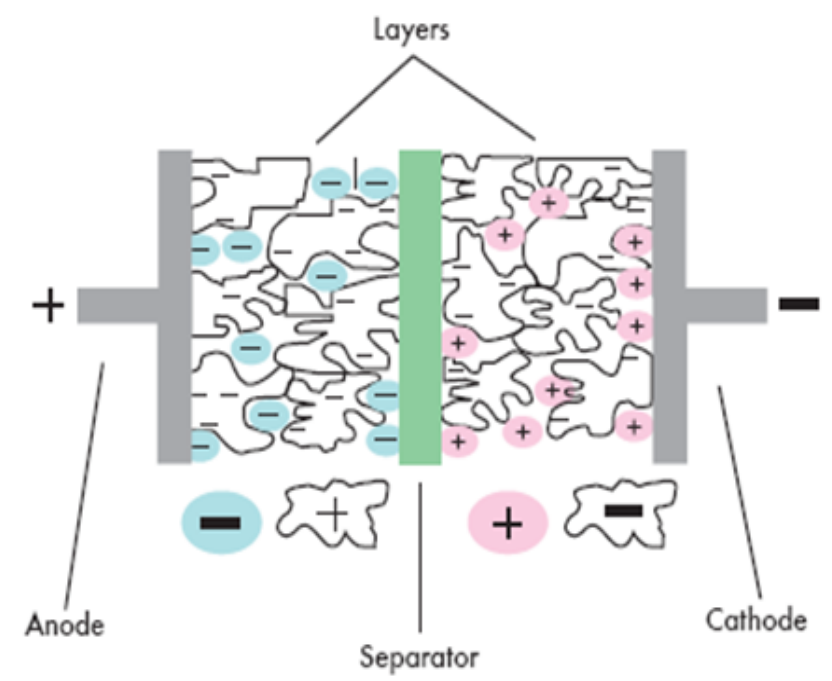

Fig. (1). Structure of double layer capacitor.

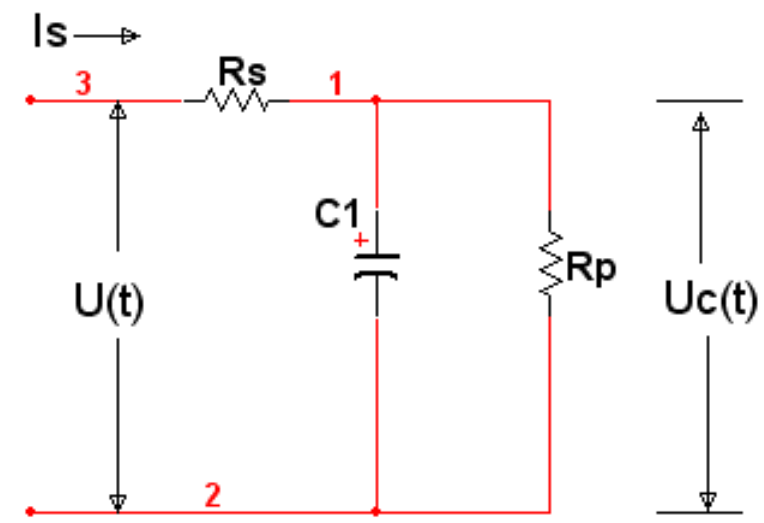

Fig. (2). Equivalent circuit diagram of super capacitor.

As with ordinary capacitor, the anode and the cathode of the layers store the positive and negative charge respectively when the super capacitor has voltage at both ends. The electrolyte will form the opposite electric charge to balance under the action of the electric field in the electrolyte and the electrode interface. This formed the electric double layer by the structure. As shown in Fig. (1), the activated carbon and the electrolyte flow closely contacting actual electrode to obtain larger contact area, thereby this increased the capacitance. In the process of discharge, charge of the anode and the cathode plate continue to leak, and the charge of contact with the electrolyte surface is reduced correspondingly. The charge and discharge process of the super capacitor does not involve a chemical reaction, and super capacitor can cycle number and has higher stability.

The internal structure of the super capacitor is very complex, Debye polarization cell model and Newman's transmission line model. But this two model is very complex when we compute. The general use of the model in the following requirements is not high in the case to the equivalent of super capacitor. It is shown in Fig. (2).

The super capacitor is equivalent to a resistor structure with an ideal capacitor $\mathrm{C}$ and resistance $\mathrm{Rp}$ are connected in parallel, and then series with Rs. Rs replaces the resistance of super capacitor produced in electrode and electrolyte. Rs replaces the resistance of static loss of super capacitor.

According to the formula:

$P=I^{2} R$

According to the formula, we can obtain the conclusion: The power consumed by Rs will be larger with current increasing when the resistance Rs is constant. Rs will product amount of heat because of energy consumption, especially obvious in the case of high current charging and discharging. So the existence of Rs has a great constraint on the super capacitor charging and discharging. 


\subsection{Analysis of Super Capacitor Energy Efficiency}

There are a lot of research on the energy efficiency of capacitor at present. We had consulted a lot of literature, but have not found a unified statement about energy efficiency of super capacitor. We believe that the energy efficiency of super capacitor has the following three kinds.

The charging efficiency $\left(\eta_{c}\right)$ is the ratio of energy storage of capacitor when super capacitor is charging and the input energy of the charger.

$\eta_{c}=\frac{E_{t}}{w_{k}}=\frac{E_{t}}{\int_{0}^{t} I u(t) d t}$

We can know from the super capacitor equivalent circuit that super capacitor internal electrolyte and plate will also produce heat because of the consumption of energy during the course of charging. The electric energy stored in the super capacitor is different from the electrical energy charged into the super capacitor.

The discharging efficiency $\left(\eta_{d}\right)$ is the ratio of energy storage $\left(E_{t}\right)$ released from the super capacitor and the energy stored in the super capacitor $\left(w_{O}\right)$.

$\eta_{d}=\frac{w_{O}}{E_{t}}$

This formula also applies to other battery. In the process of discharging a part of energy will be consumed in the form of heat. Both the chemical battery or super capacitor Can't 100 percent release of stored energy because of discharging voltage constraints.

The efficiency of energy storage $\left(\eta_{e}\right)$ is the ratio of energy storage released from the super capacitor $\left(w_{O}\right)$ and the energy stored in the super capacitor $\left(w_{i}\right)$.

$\eta_{e}=\frac{w_{O}}{w_{i}}$

The efficiency of energy conversion mainly refers to ratio of the capacitor energy released and the charger charge energy. It represents the utilization efficiency of which the electrical energy output.

$\eta_{t}=\frac{w_{O}}{w_{k}}$

The efficiency of energy storage is different from the charging efficiency. Although efficiency of energy storage is definited of charging efficiency in some literature, but the efficiency of energy storage include the process of charging, discharging and stewing. There is little loss of energy in the process of charging and discharging. There is loss of energy in process of stewing because of the existence of $\mathrm{Rp}$ from the equivalent structure diagram of super capacitor. If the efficiency of loss energy is smaller than 4 percent, efficiency of energy storage can be approximately regarded as the product of charging and discharging efficiency.

$\eta_{e}=\eta_{c} \times \eta_{d}$

The efficiency of energy conversion depends on the efficiency of charging/discharging and stewing. The efficiency of energy conversion will be higher with the increase of the efficiency of charging/discharging and stewing.

\section{ANALYSIS OF CHARGING EFFICIENCY WITH DIFFERENT CHARGING MODE}

The charging process of the super capacitor is the physical process not involve chemical reactions, This makes the super capacitor has little effect on aging in the use of the process of charging or discharging. Super capacitor can realize the fast charge without memory effect. In theory, the charge of super capacitor is unlimited. According to the characteristics of super capacitor, charging mode of super capacitor include constant-current charging, constant-voltage charging and constant-power charging. The different charging mode has great influence on charging efficiency of super capacitor. In practical application, we select the super capacitor according to the specific situation .

\subsection{Constant-current Charging}

Super capacitor charging process of the equivalent circuit can be simplified as shown in Fig. (3). In the equivalent circuit diagram,it is simplified as an ideal capacitor and a resistor in series circuit. Its resistance is an important factor to affect the charging time and charging efficiency.

The constant-current charging is a charging method widely used at present. It can be set different charging current according different demands. The charging will be shorter when the charging current become larger. In Fig. (3), assuming I charging current, charging voltage of $U(t)$, the initial capacitor voltage $U_{O}$ is 0 .We can calculate the charging efficiency in the below formula.

$\eta_{c}=\frac{1}{1+\frac{2 R_{S} C}{T}}$

It can be seen in formula(6), Rs and $\mathrm{C}$ is constant values of super capacitor, so the charging efficiency is only relevant for the charging time, the charging efficiency will be higher when the charging time become longer. The calculation of the charging time can be simple expressed as shown in the below formula.

$T=\frac{C \times U_{C}(T)}{I(t)}$

$\mathrm{U}_{c}(T)$ is a constant value of rated voltage of super capacitor. From the formula we can see that the charging current 


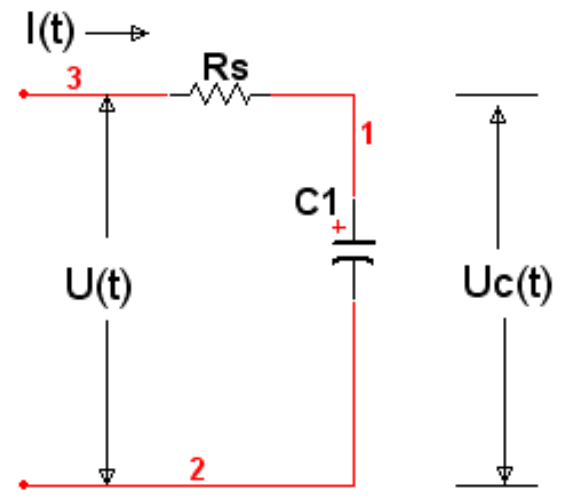

Fig. (3). Super capacitor equivalent simplified circuit diagram.

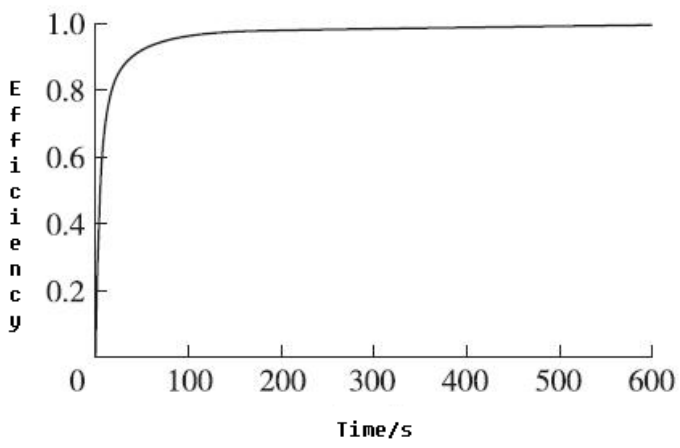

Fig. (4). Relationship of charging efficiency and charging time.

and the charging time is inversely proportional and it is independent of other factors. We had calculated the charging efficiency In the $3500 \mathrm{~F} / 2.7 \mathrm{~V}$ of super capacitor as an experiment. In this experiment, the capacitance $\mathrm{C}=3500 \mathrm{~F}$, $R s=0.5 \mathrm{~m} \Omega$, the initial voltage is $0 \mathrm{v}$, the rated voltage is $2.7 \mathrm{v}$. The relationship between the charging efficiency and charging time is shown in Fig. (4) [11].

As it can be seen from Fig. (4), if the charging time is within $50 \mathrm{~s}$ then the charging efficiency reached $90 \%$ at the most, prolong the charging time to $200 \mathrm{~s}$, the charging efficiency can reach above $95 \%$. The charging efficiency is higher with much time. We have to have a balanced consideration of charging current and charging time o in the super capacitor charging, so as to achieve the purpose of quick and efficient.

\subsection{Constant-voltage Charging}

The constant-voltage charging refers to keep constant voltage charging power supply unchanged in the process of charging for super capacitor, the current will continue to decrease constantly with the charge processing. When we select the constant-voltage charging mode we must select suitable voltage source, and the voltage pulsation must be little.
According to Fig. (3) we can draw that:

$U=i(t) \times R_{s}+U_{c}(t)$

By the above formula, we can get the output voltage of super capacitor.

$U_{c}(t)=U+\left(U_{C O}-U\right) \times e^{-\frac{t}{R C}}$

Among them, $U_{C O}$ is the super initial capacitor voltage, this experiment provided the initial voltage of 0 . The energy consumed by resistor is $W_{R}$, the capacitor charging energy is $W_{C}$, the charging time is $\mathrm{T}$. We can obtain the formula as below.

$W_{R}=i^{2}(t) \times R_{s}=\frac{1}{2} U^{2} C\left(1-e^{-\frac{2 T}{R_{s} C}}\right)$

$W_{C}=\frac{1}{2} U^{2} C\left(1-e^{-\frac{T}{R_{s} C}}\right)^{2}$

By formula (2) and formula (3) we can obtain the formula of calculation of the charging efficiency. 


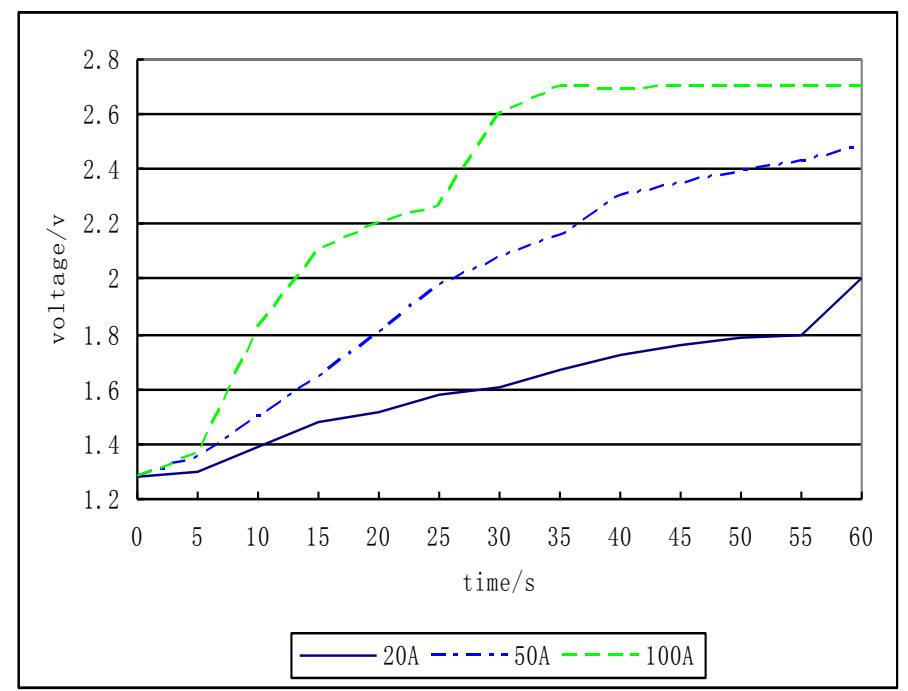

Fig. (5). The voltage change of constant current charging.

$\eta_{c}=\frac{W_{C}}{W_{C}+W_{R}}=\frac{1}{2}\left(1-e^{-\frac{T}{R_{S} C}}\right)$

It can be seen from the above equation that the charging efficiency in constant voltage charging process increases with the increasing of charging time, it can reach $50 \%$, the low efficiency of constant-voltage charging associated with the charging mode. Due to the characteristics of super capacitor caused by the super capacitor is not suited for the constant-voltage charging method to charge. But different from the conventional battery charging, the charging efficiency disconnected with resistance for the same super capacitor.

\subsection{Constant-power Charging}

In certain circumstances, we will select the constant power supply for charging. The advantage of constant-power charging is it can not affect other circuit and have constant input power. In this charging mode the charging voltage and current changes changed with time changes [3].

$P=i^{2}(t) \times R+U_{C}(T) \times i(t)$

Further derivation, we can calculate the current:

$i(t)=\frac{-U_{C}(t)+\sqrt{U_{C}^{2}(t)+4 R_{S} \times P}}{2 R_{S}}$

For the convenience of calculation, the super capacitor terminal voltage of $U_{C 0}$ to $U_{C}(T)$ is divided into $\mathrm{N}$ parts, which considers each equal parts of current is constant, set the appropriate step size, and then calculated through the differential,we can get the constant-power efficiency formula of constant power for charging:

$\eta=\frac{\frac{1}{2} C\left(U_{C}^{2}(T)-U_{C 0}^{2}\right)}{P \times t}$

Analysis of above formula can be obtained that the charge energy is mainly consumed in the resistance of Rs at the beginning, with the increase of the voltage across the capacitor and the decreases of charging current, the energy consumed by heat also decreases, the charging efficiency gradually improved, finally can reach more than $90 \%$. The constant-power charging applicated in the photovoltaic power generation.

Combination of the above theoretical analysis, it is not difficult to find that there is great correlation of super capacitor charging efficiency and charging method, which has great relationship with the super capacitor itself. The efficiency of constant-current charging is high, but by the late voltage of both ends of the capacitor is too large, the efficiency of the constant voltage charging become too lower at this time. The control circuit of constant-power charging complicated, so we can take way of combined charging. It will take a large current charging mode when the super capacitor terminal voltage low, with increasing charging terminal voltage change for decreasing current or constant voltage mode, so that the charging more fully.

\section{VERIFICATION OF THE CHARGING EFFICIEN- CY}

The size and direction of current will change at the end of constant-current charging and discharging, so we can measure the equivalent series resistance of super capacitor through the current step method. The specific method is accurate to record the change of voltage of super capacitor when the size and direction of current changed. Using the following relation:

$E S R=\frac{U}{I}$

At temperature of 25 degrees Celsius, we use $2.7 \mathrm{~V}$ as the upper limit voltage of the super capacitor whose capacitance is $3700 \mathrm{~F}$, and use $1.3 \mathrm{v}$ as the lower limit voltage. The super capacitor was charged with constant current I, its value is 20A, 50A, 100A.

It shows that the variation of the charging process of the voltage of super capacitor in Fig. (5). It has a sinuate margin obviously at the beginning and the end of charging. The 


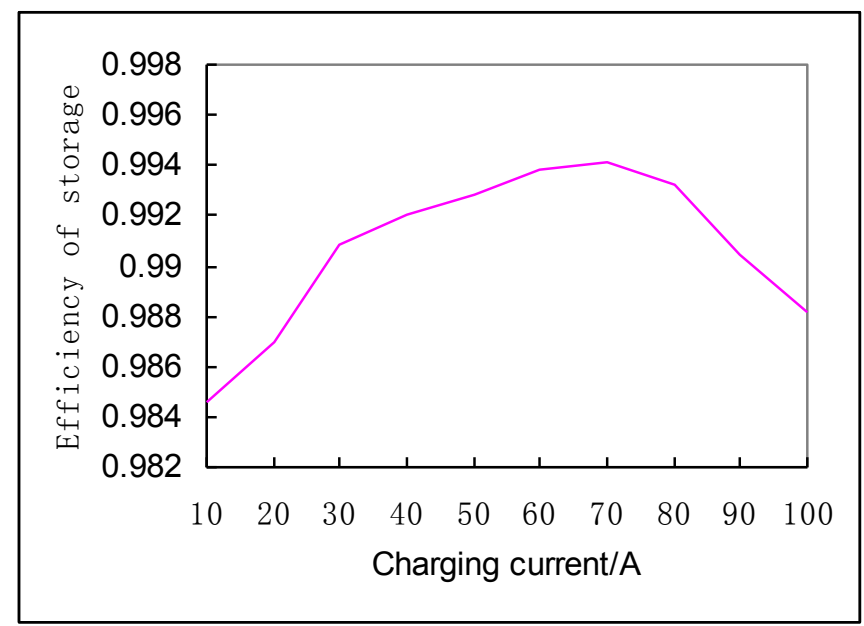

Fig. (6). Relationship of efficiency and current.

charging voltage of Super capacitor changes linearly. The voltage changes quickly in the initial stage, and changes gently in the middle, in the end it change quickly again. This proved that: The time of super capacitor charged fully is shorter when charging current get larger. Causes of terminal voltage fluctuation of super capacitor is mainly affected by the impact of the charging current and the equivalent series resistance. The effects of the two factors make the effective energy storage of super capacitor changes, it leads effective energy storage to lower with the increase of charging current.

We have tested the charging efficiency with the constant charging current.

From the Table $\mathbf{1}$ we can know the efficiency can reach above 0.99. The charging efficiency is most high when charging current is 70A. The analysis as shown in Fig. (5).

Table 1. Efficiency of different charging current

\begin{tabular}{|c|c|c|}
\hline Id & Charging Current/A & Efficiency \\
\hline \hline 1 & 10 & 0.9846 \\
\hline 2 & 20 & 0.987 \\
\hline 3 & 30 & 0.9908 \\
\hline 4 & 40 & 0.992 \\
\hline 5 & 50 & 0.9928 \\
\hline 6 & 60 & 0.9938 \\
\hline 7 & 70 & 0.9941 \\
\hline 8 & 80 & 0.9932 \\
\hline 9 & 90 & 0.9904 \\
\hline 10 & 100 & 0.9882 \\
\hline
\end{tabular}

As shown in Fig. (6), describes the change of the relationship between charging efficiency and charging current in the condition of constant current. The charging efficiency is lower with the current i smaller, it gradually increase with the increase of charging current. When the charging current increase to a certain value, the charging efficiency begin to reduce. So in the choice of charging current, in order to meet the requirements of users and achieve optimal configuration of super capacitor, we should consider the relations of the charging time, energy storage and charging efficiency.

\section{CONCLUSION}

This paper gives a clear definition charging efficiency, discharging efficiency and storage efficiency of super capacitor. Through the calculation of charge efficiency of the three charging modes include constant-current charging, constant-voltage charging and constant-power charging. We can draw that the constant current charging can realize fast charging, The charging efficiency can be up to $95 \%$ or more, it is the most suitable for super capacitor charging. We choose the charging current according to the actual situation because the charging efficiency is inversely proportional to charging current.

Characteristics of super capacitor and constant-voltage charging mode determines the constant voltage charging efficiency is less than $60 \%$, but this can't represent the efficiency of super capacitor is low, only the constant-voltage charging mode is not suitable for charging of super capacitor. In the choice of charging mode it can also be combined with constant current and constant voltage in two ways, charge with constant-current at the beginning, when the voltage reach nearly rated voltage, then charge with constant-voltage.

Constant-power charging is generated for photovoltaic power generation based on MPPT, the theoretical efficiency can reach as high as $95 \%$, but the constant power source to achieve more complex, it is not suitable for a large number of application. We must choose the proper charging mode according to the different needs. 


\section{CONFLICT OF INTEREST}

The authors confirm that this article content has no conflicts of interest.

\section{ACKNOWLEDGEMENTS}

This paper was supported by Professor Gao Jian. My family also helped me a lot during the course of my researching. Thanks for their selfless help.

\section{REFERENCES}

[1] J.M. Blanes, R. Gutierrez, A. Garrigos, J.L. Lizan, and J.M. Cuadrado, "Electric vehicle battery life extension using ultra-capacitors and an FPGA controlled interleaved buck-boost converter" IEEE Transactions on Power Electronics, vol. 28, no.12, pp. 5940-5948, 2013.

[2] M. Steiner, M. Klohr, and S. Pagiela, "Energy storage system with ultra-caps on board of railway vehicles", European Conference on Power Electronics and Applications, Aalborg, Denmark, 2007.

[3] Y. Meng, S. Zhang, J. Chen, M. Duan, "The Impact of Charging Mode Pairs of Super Capacitor Energy Efficiency", Chinese Journal of Electron Devices, vol. 37, no. 1, pp. 13-16, 2014.
[4] M. Uno, and A. Kukita, "Double-switch equalizer using parallel-or series-parallel-resonant inverter and voltage multiplier for seriesconnected super-capacitors" IEEE Transactions on Power Electronics, vol. 29, no. 2, pp. 812-828, 2014.

[5] Q. Xu, and H. Bian, "EDLC Charging Performance forMicrogrid Application" Journal of Southeast University, vol. 26, no. 3, pp 415-420, 2010.

[6] Z. Yu, and J. Zhenhua, "Dynamic power sharing strategy for active hybrid energy storage systems," Vehicle Power And Propulsion Conference(VPPC), pp. 558-563, 2009.

[7] H. Farsi, and F. Gobal, "Artificial neural network simulator fou supercapacitor performance prediction"Computational Materials Science, vol. 39, no. 3, pp. 678, 2007.

[8] J. Nan, J. Wang, and F. Sun, "Study of energy management system of electric vehicle," Transactions of Beijing Institute of Technology, vol. 25, no. 5, pp. 384-389, 2005.

[9] S. Wang, T. Wei, and Z. Qi, "Energy saving system based on supercapacitor," In: Proceedings of the CSEE, vol. 30, no. 9, pp. 105, 2010 .

[10] S. Mao, and X. Cai, "Control strategy for power conditioning system of large capacity cascaded battery energy storage system," In: Power System Technology, vol. 36, no. 9, pp. 226-231, 2012,.(in chinese)

[11] A. Tani, M. B. Camara, and B. Dakyo, "Energy management based on frequency for hybrid electric vehicle applications;fuelcell/lithium-battery and ultra-capacitors," IEEE Transactions on Vehicular Technology, vol. 61, no. 8, pp. 3375-3386, 2012.

Received: June 02, 2015

Revised: August 02, 2015

Accepted: September 05, 2015

(C) Zhang Dedi; Licensee Bentham Open.

This is an open access article licensed under the terms of the Creative Commons Attribution Non-Commercial License (http://creativecommons.org/licenses/by$\mathrm{nc} / 3.0 /$ ) which permits unrestricted, non-commercial use, distribution and reproduction in any medium, provided the work is properly cited. 